\title{
Versuch einer Charakteristik der ungarischen Sprache
}

In diesem Beitrag können wir kein vollständiges Bild von dem Bau der ungarischen sprache zeichnen; dazu wäre ja nur eine wissenschaftlich deskriptive Grammatik imstande. Wir wollen lediglich danach streben, einige charakteristische Züge der Laut-, Wort- und Satzstruktur auf Grund teilweise neuer Gesichtspunkte aufzuzeigen.

\section{Die I autstruktur'1}

1. Vokale. Der Vokalbestand der ungarischen Sprache kann in folgendem Schema zusammengefasst werden, wenn man die Quantität, die Bewegungen der Zunge in waagerechter und senkrechter Richtung, sowie die Art der Lippentätigkeit in Betracht zieht (Tabelle 1).

Wie die Tabelle deutlich zeigt, ordnen sich die Vokale auf Grund der physiologischen Eigentümlichkeiten ihrer Bildung einerseits in gleichartige Reihen, andererseits in gegensätzliche Paare, sie bilden also ein geschlossenes System. Nur vier Vokale stehen halb und halb) ausserhalb des Systems, weil ihnen - wohl im Ergebnis der historischen Entwicklung eine Entsprechung fehlt. In der heutigen ungarischen Gemeinsprache steht neben dem langen $a$ kein kurzes $a$, dem kurzen $e$ fehlt das ihm entsprechende lange $\bar{e}$. Ferner hat das kurze $a$ kein langes Paar $\bar{a}$ und das lange $e ́$ kein kurzes $\ddot{e}$. Das Sprachbewusstsein hat jedoch die allein gebliebenen Laute zu Ge-

1 Die Fragen der Lautstruktur der ungarischen Sprache behandle ich ausführlicher in meiner Arbeit "Hangtan" [Lautlehre]: Magyar Nyelvjárások V 42-101 und Sonderabdruck: Debrecen 1959. 
Tabelle 1 .

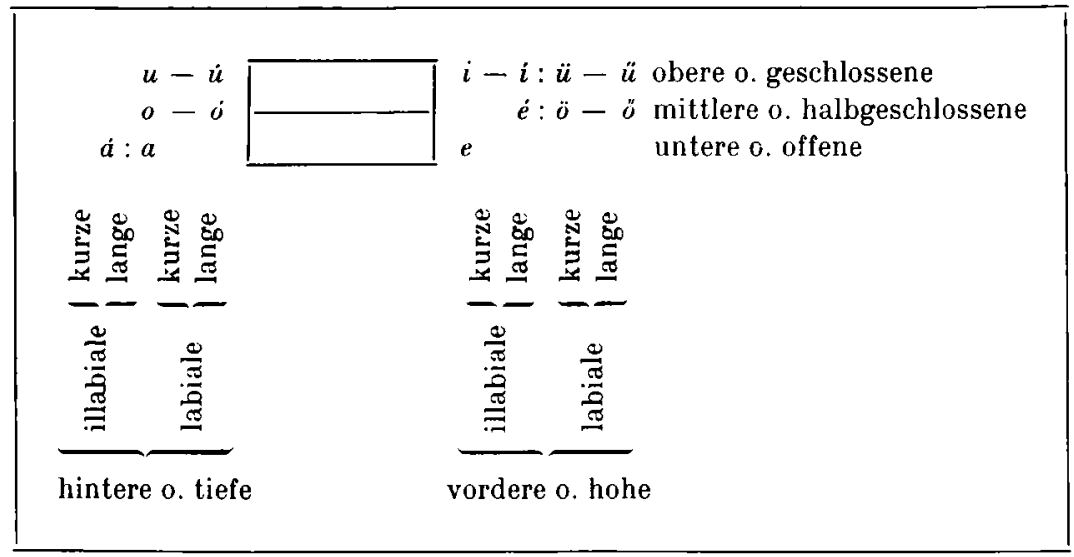

gensätzen gepaart und das folgende phonologische System der Vokale ausgebildet (Tabelle 2).

In diesem System ist der Gegensatz auf Grund der Quantität am vollkommensten ausgebildet: jeder kurze Vokal hat eine lange Entsprechung. Doch gibt es zwischen den einzelnen Gegensatzpaaren bestimmte Wertunterschiede. Am klarsten bestimmt ist der Gegensatz bei den Paaren unterer Zungenstellung $a-a ́$ und $e-\dot{e}$, weil hier die Differenz der Quantität auch durch eine hochgradige Differenz der Qualität verdeutlicht wird: das $a$ ist nämlich labial, sein langes Paar $a ́$ hingegen illabial. Andererseits ist das $e$ offen, das lange $e ́$ hingegen halbgeschlossen. Kein Wunder, wenn hier weder Schwankungen in der Aussprache noch Schwierigkeiten in der Rechtschreibung auftreten und die Sprache diese lautlichen Gegensätze in grossem Masse ausnutzen kann zur Differenzierung von Wortbedentungen, Satzverhältnissen und stilistischen Werten, z.B.

Tabelle 2.

\begin{tabular}{|c|c|}
\hline $\begin{array}{l}u-\dot{u} \\
o-\dot{o} \\
a-\dot{a}\end{array}$ & $\begin{array}{l}i-i: \ddot{u}-\ddot{u} \\
e \ddot{o}-\dot{o} \\
e-\dot{e}\end{array}$ \\
\hline
\end{tabular}


in solchen Wortpaaren wie: kar 'Arm' -- kár 'Schade', keret 'Rahmen' - kéret 'lässt bitten'; várna 'er würde warten' cárná 'er würde ihn erwarten', gyermeke 'sein Kind' - gyermeké 'das des Kindes'; ad - ád 'er gibt', mitevö - mitérö 'was tun'. Die Oppositionen mittlerer Zungenstellung $o-\dot{o}, \ddot{o}-\ddot{o}$ zeigen neben Quantitätsdifferenzen auch solche der Qualität, die aber gering sind. Das lange $o$, ô zeigt nämlich eine Zungenstellung, die nicht um einen granzen, sondern nur um einen halben Grad höher ist als bei ihren Paaren o, o. Die Schwankung der Aussprache hat hier zwar schon etwas Spielraum, doch benützt die Sprache auch diese Lautgegensätze zur Differenzicrung von Bedeutungen, z.B. kor 'Zeitalter' - kór 'Krankheit', örült 'er freute sich' - örült 'wahnsinnig'; ovoda - óvoda 'Kindergarten'. Die Quantitätsdifferenz von $u-\dot{u}, i-i, \ddot{u}-$ ¿ oberer Zungenstellung hingegen wird durch keinen wahrnehmbaren Qualitätsunterschied verstärkt. Das ist der Grund dafür, dass die Schwankungen der Aussprache hier beachtliche Ausdehnung haben und die Bezeichnung der Lautdauer eben bei den Vokalen oberer Zungenstellung eine der schwierigsten Fragen der ungarischen Orthographie ist. Die Korrelation "kurz - lang" der Vokale mit hoher Zungenstellung ist als solche nicht geeignet, Bedeutungen zu differenzieren; darum kommt sie nur - mit Ausnahme vereinzelter Fälle - bei einigen Wortfamilien als Stammwechsel vor: ur 'Herr', úmal: '(dem) Herrn', uri 'herrisch' - urat '(den) Herrn', urak '(die) Herren', uradalom 'Herrschaftsgut'; ir 'er schreibt', irok 'ich schreibe', irtam 'ich schrieb' - irat 'Schrift', irodalom 'Literatur'; tüz 'Feuer', tüzzel 'mit Feuer', tüzröl 'von Fener weg' tiizet 'Feuer (Akk.)', tüzek '(die) Fener', tüzes 'feurig'.

Die Anzahl der Vokalunterschiede palatal - velar beläuft sich nur auf sechs: $a-e, \dot{a}-\dot{e}, o-\ddot{o}, \dot{o}-\ddot{o}, u-\ddot{u}, \dot{u}-\ddot{u}$. Allein, ohne velare Entsprechung steht in der Reihe der pilatalen Vokale vor allem das $i, i$, ausserdem aus historischen Gründen ein solches $e$, das aus geschlossenerem $\ddot{e}$ entstanden ist, endlich - mit Rücksicht auf seine ursprüngliche physiologische Natur - bis zu einem gewissen Grade auch das é. Darum pflegt man diese vier palatalen Vokale als indifferent zu bezeichnen. 
Die Korrelation "palatal - velar" benutzt die ungarische Sprache erstens bei der Erscheinung der Vokalharmonie. Die Vokalharmonie besteht darin, dass in einfachen ungarischen Wörtern entweder lauter palatale oder lauter velare Vokale stehen, die vier indifferenten Vokale aber auch mit velaren Vokalen im Worte stehen können. Wörter mit Vokalen der palatalen Reihe sind z.B. ember 'Mensch', pöröl 'er streitet'; Wörter mit velaren Vokalen: család 'Familie', háború 'Krieg'; gemischter Vokalismus: ital 'Trank', kieán 'er wünscht', leány (<lëány) 'Mädchen', méla 'träumerisch'. Infolge der Vokalharmonie sind im Ungarischen Formantien mit zwei, drei und einer Form entstanden. Die zweiförmigen Formantien schliessen sich an Stämme mit Vokalen der palatalen Reihe in ihrer vordervokalischen Form an, an Stämme mit velarem und gemischtem Vokalismus in ihrer hintervokalischen Form. So z.B. das Kasussuffix -ban/-ben: liertben 'im Garten', házban 'im Haus', italban 'im Getränk'; das Bildungssuffix der Substantiva -ás/-és: liérdezés 'das Fragen', olvasás 'das Lesen', tanitás 'das Lehren'; das Bildungssuffix des Partizips -ó/-î: keresö-'suchend', álló 'stehend', kivánó 'wünschend'. Die Formantien mit drei Varianten haben eine hintervokalische Form und zwei vordervokalische, die letzteren passen sich auch der Lippentätigkeit nach der Qualität des Stammrokalismus an. Z.B. das Kasussuffix -hoz/-hez/-höz: házhoz 'zum Haus', kézhez 'zur' Hand', földhöz 'zur Erde'. Die Formantien mit nur einer Variante haben einen indifferenten Vokal, also tritt hier keine Vokalharmonie auf, z.B. -ni in der Bildung der Infinitivform: járni 'gehen', kérni 'bitten', kívánni 'wünschen'; oder das Bildungssuffix der Verben -it: tanit 'lehren', épit 'bauen'; oder das Besitzzeichen -é: államé 'das des Staates', emberé 'das des Menschen'.

Die Vokalharmonie ist eine Assimilationserscheinung. Sie hat im ungarischen Lautsystem einerseits Eintönigkeit, andererseits aber auch Mannigfaltigkeit hervorgerufen. Eintönig ist der lautliche Aufbau der ungarischen Wörter, da ja Würter wie embertelenségében 'in seiner Unmenschlichkeit' oder állhalallanságában 'in seiner Unbeständigkeit' nicht zu den Seltenheiten gehören. Doch verursachen die zwei- und droiförmigen 
Formantien bei den Wörtern derselben Funktion auch eine gewisse Mannigfaltigkeit, z.B. embertelenségében: állhalallanságában; kapdos 'hin- und hergreifen': nyeldes 'nacheinander verschlingen': öldös 'oftmals morden'. Ausserdem erfüllt die Vokalharmonie eine wichtige Sprachfunktion, indem sie die Einheit der Wortbedeutung sichert. Dass der einheitliche Bedeutungsinhalt des Wortes nicht in seine Bestandteile zerfällt, wird vorzüglich durch den Ansgleich der Qualität der Vokale gesichert.

Den Gegensatz palatal - velar benutzt die sprache ausserdem auch zur Differenzierung von Bedeutungen. Sie bezeichnet durch den Kontrast des vorderen und des hinteren Vokals die Gegensätzlichkeit von Nähe und Ferne oder den Unterschied der Intensität. Die Abart mit palatalen Vokal bezeichnet die Nähe oder einen niedrigen Grad der Intensität, während diejenige mit velarem Vokal die Ferne oder einen höheren Grad der Intensität angribt. Manchmal ändert sich auch die Grundbedentung mit der verschiedenen Lautqualität vollständigr. Den Unterschied der Entfernung bezeichnen wir mit den zwei verschiedenen Formen des Demonstrativpronomens ez 'dieser' - $a z$ 'jener'. Die kleinere oder grössere Intensität derselben Erscheinung drücken Wortpaare aus, wie csett-csatt 'piff, paff!', önt 'giessen' - ont 'strömen', kever 'mischen' -Lavar 'quirlen', döbben 'er wird betroffen' - dobban 'es pocht', püffen 'es plumpst' - puffan 'es pufft'. Vollständige Veränderung der Grundbedeutung zeigen: cseléd 'Dienstmagd' - család 'Familie', tüzök 'ich stecke' — túzok 'Trappe'.

Der Gegensatz labial - illabial ist in der Lautstruktur des Ungarischen am wenigsten ausgebildet. Die hinten gebildeten Vokale haben nämlich - mit Ausnahme des einzigen á alle Lippenrundung, sind also labial, so bietet sich nur bei den vorderen Vokalen die Möglichkeit für den Gegensatz labial illabial. Auch hier kommen zuerst die kurzen Gegensatzpaare in Betracht; besonders $i$ : $\ddot{u}$ und noch mehr der Gegensatz $e$ : $\ddot{o}$ haben mundartliche Varianten bzw. Wortpaare von stilistischem Wert in grosser Anzahl hervorgerufen, z.B. idó 'Zeit' : üdö, fil : fül 'Ohr', seper 'er fegt' : söpör, fel 'herauf' : föl.

Nach dem Grade der Geschlossenheit der Vokale können im 
Ungarischen grleichfalls Abwandlungen vorkommen. Besonders wichtig ist das mannigfaltige Verhalten des stammauslautenden kurzen Vokals ror den verschiedenen Formelementen. Im Konjugationssystem des Verbs hall 'hören' erscheint der stammauslautende Vokil in drei Variationen: hallu-nk 'wir' hören', hallo-k 'ich höre', halla-nak 'sie hören'. Dasselbe grilt für die Deklination des Nomens ház 'Haus': házu-nl: 'unser Haus', házo-n 'auf dem Haus', háza-tok 'ener Haus'. Zwei Variationen kommen ror beim Verb äl sitzen' oder beim Nomen föld

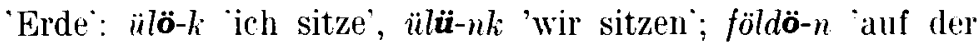
Erde', földü-nk 'unsere Erde". Im Stamminlaut kommt eine ähnliche Variation vor z.I3. beim Verb ran 'ist' : colt 'war', ferner in der Wortfamilie der Demonstrativpronomina $\boldsymbol{a}_{z}$ 'jener' : ott 'dort' : úgy 'auf jene Weise'; $\boldsymbol{e} z$ 'dieser' : itt 'hier' : ig!y 'auf diese Weise'. Sonst sind die Unterschiede des Grades der lieschlossenheit nicht geeignet. Bedeutungen zu unterscheiden.

2. Konsonanten. Die kurzen ungarischen Konsonanten können nach Stimnbeteiligung, Artikulationsstelle und Artikulationsart in folgende Reihen ringeteilt werden (Tabelle 3):

Tabelle 3.

\begin{tabular}{|c|c|c|c|c|c|c|c|c|c|}
\hline & \multicolumn{2}{|c|}{ 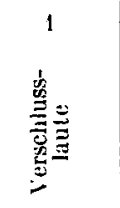 } & \multicolumn{2}{|c|}{ 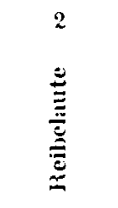 } & \multicolumn{2}{|c|}{ 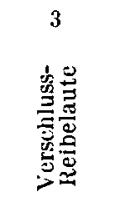 } & 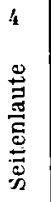 & 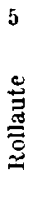 & 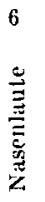 \\
\hline & 哭 & 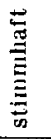 & 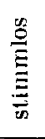 & 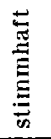 & 骂 & 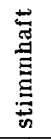 & 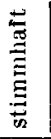 & 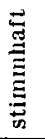 & 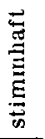 \\
\hline I Lippenlaute & $p$ & $b$ & & & & & & & $m$ \\
\hline II Lippen-Zahnlaute & & & $f$ & ' & & & & & \\
\hline III Zahnlaute & $t$ & $d$ & $\begin{array}{l}s z \\
s\end{array}$ & $\begin{array}{l}z \\
z s\end{array}$ & $\begin{array}{l}c \\
c s\end{array}$ & $\begin{array}{l}d z \\
d z s\end{array}$ & $l$ & $r$ & $n$ \\
\hline IV Zahn-Gaumenlaute & & & & & $t y$ & gy & ly & & $n y$ \\
\hline V Gaumenlaute & $k$ & $g$ & $\dot{x}$ & $i$ & & & & & $\eta$ \\
\hline VI Kehllaut & & & $h$ & & & & & & \\
\hline
\end{tabular}


In diese Tabelle haben wir lediglich einen solchen Laut aufgenommen, der nicht mehr der Gemeinsprache angehört, d.i. das $l y$. Es wird in der Schrift allerdings beibehalten, sonst aber spricht man im grössten 'Teil des ungarischen Sprachgebiets ein $j$ statt $l y$. Die stimmlose Entsprechung von $j$, d.i. $\dot{\chi}$, ist zwar in der Sprache vorhanden, da sie aber nur in einer bestimmten Position rorkommt (lépj 'tritt!', rakj 'lege!', döfj 'stoss!'), nimmt das ungarische Sprachgefühl davon keine Kenntnis, und auch die Orthographie bezeichnet es nicht. Geradeso unterscheidet sich auch der Palatal-, bzw. Velarlaut $\eta$ vom Zahnlaut $n$, doch erscheint er im Sprachbewusstsein der ungarischen Sprecher nicht als besonderer Laut, weil er nur in bestimmten Lautsituationen vorkommt, nämlich vor $k$ und $g$ (senki 'niemand', harang 'Glocke').

Praktisch genommen hat jeder kurze Konsonant eine lange Entsprechung, d.h. die Quantitätskorrelation ist im ungarischen Konsonantensystem vollkommen ausgebildet. Die kurzen langen Konsonantenpaare werden in der ungarischen Sprache in starkem Ausmass zum Ausdruck lexikalischer und syntaktischer Unterschiedlichkeiten ausgenutzt, doch sind auch die nur stilistischen Ausdrucksvarianten in ziemlich grosser Anzahl vorhanden, z.B. hal 1. 'Fisch', 2. 'er stirbt' - hall 'er hört', épen 'unversehrt' -- éppen 'eben', szemel 'er liest aus' - szemmel 'mit Auge', anya 'Mutter' - annya (geschrieben: anyja) 'seine o. ihre Mutter' (lexikalische und syntaktische Kontraste); egyetlen - eggyetlen 'einzig', ahoz - ahhoz 'dazu', erős -- erőss 'stark' (stilistische Varianten).

Deutlich ausgebildet ist in der ungarischen Sprache der Gegensatz stimmlos - stimmhaft, obwohl sich derselbe nicht auf alle Konsonanten gleichmässig erstreckt. Ausser den Konsonanten mit zwei Variationen (z.B. $p-b$ ) gibt es im ungarischen Lautsystem auch Konsonanten mit nur einer Variation: das stimmlose $h$ und die stimmhaften $l, r, m, n, n y$. Ein Gegensatz von stimmlos und stimmhaft kann natürlich nur bei den Konsonanten mit zwei Variationen vorkommen. Dieser Lautgegensatz hält Bedeutungen auseinander: z.B. por 'Staub' bor 'Wein', hat 'sechs' - had 'Heer', lép 'Bild' - gép 'Maschine', sir 'er weint' - zsir 'Fett'; kenyeret 'Brot (Akk.)' kenyered 'dein Brot', várt 'er wartete' - várd 'warte auf ihn!'. 
Assimilation nach dem Stimmton ist jenes Gesetz der ungarischen Sprache, das in der Einheit des Wortes oder des Redetaktes den Gegensatz unmittelbar aufeinander folgender Konsonanten mit und ohne Stimmton (stimmhaft - stimmlos, stimmlos - stimmhaft) auflöst und dadurch den Gang der Lautbildung glatter und frei von Anstössen gestaltet. Die Assimilation nach dem Stimmton wirkt regressiv: das zweite Glied der Konsonantenverbindung wirkt auf das erste und assimiliert es in der Richtung des entsprechenden Lautgegensatzes, d.h. macht es stimmlos oder stimmhaft. Z.B. wirkt im Worte életben 'im Leben' das stimmhafte $b$ auf das vorangehende stimmlose $t$ ein und verändert es in der Richtung des Gegensatzpaares $t-d$ zu einem stimmhaften $d$, also: éledben. In einem Wort wie adhat 'er kann geben' löst sich der Gegensatz des stimmhaften $d$ und des stimmlosen $h$ so auf, dass das stimmlose $h$ das vorangehende stimmhafte $d$ in der Richtung des Gegensatzpaares $t-d$ zum stimmlosen $t$ verändert, d.h. die Aussprache des Wortes lautet: athat.

Natürlich hommt die Assimilation nicht in jeder Relation der Konsonanten zu gleicher Geltung. Nur die Konsonanten, die in bezug auf die Stimmbeteiligung als Oppositionen zusammengehören, werden von dieser Gesetzmässigkeit betroffen. Z.B. életben : éledben, wo das $t$ assimiliert wird und das $b$ assimilierend ist. Hingegen wirkt in der Form dobtam : doptam 'ich habe geworfen' das $\iota$ assimilierend, während $b$ assimiliert wird. Es gibt hier nur eine Ausnahme. Das v besitzt nämlich, obwohl es das $f$ als Gegensatzpaar neben sich hat, keine assimilierende Wirkung. So lautet z.B. die Aussprache des zusammengesetzten Wortes tévtan 'Irrlehre' wie téftan, hingegen entspricht die gemeinsprachliche Lautung des Wortes ötven 'fünfzig' der etymologischen Schreibweise, also nicht etwa ödven. Demgegenüber ist es natürlich, dass das $h$ als stimmloser Konsonant mit einer Variation nur assimilierend wirkt, selbst aber keine Assimilation erleidet, wie wir im Worte adhat anstatt $d$ unter dem Einfluss des $h$ ein $t$ aussprechen: athat. Bemerkenswert ist, dass die stimmhaften Konsonanten mit einer Variation (also $l, r, m, n, n y$ ) nicht nur keiner assimilatorischen Wirkung unterliegen, d.h. nie stimmlos werden können (dies ist natürlich, da sie keine stimmloseu Entsprechungen aufweisen), 
sondern sie haben auch keine assimilierende Fähigkeit, d.h. sie verwandeln den vorhergehenden stimmlosen Konsonanten nicht in einen stimmhaften. So zeigt sich z.B. weder im Worte lelkes 'begeistert', noch im Worte ineklö 'singend' eine Assimilation.

Nach der Artikulationsstelle haben sich inı ungarischen Konsonantensystem sechs Reihen entwickelt (vgl. Tabelle 3). Es ist klar, dass eine solche Skala mit sechs Reihen nicht geeignet ist, Gegensatzpaare zu schaffen und so Bedeutungen auseinanderzuhalten. Die Gesetzmässigkeit der Assimilation zeigt sich aber auch hier, obwohl die Assimilation nach der Artikulationsstelle bei weitem keine so allgemein wirkende ist als bei der des Stimmtones. Nach der Stelle der Lautbildung assimiliert z.B. im Wort különb '(jemandem) überlegen' das bilabiale $b$ den vorhergehenden dentalen Nasal, also $n>m$ : külömb. Ferner löst der Mechanismus der Aussprache auch den Gegensatz nach der Stelle der Lautbildung durch das fiesetz der Verschmelzung. Das besteht darin, dass wir anstatt der Verbindung des dentalen $t, d, n$ und des darauffolgenden Gaumen$j$ einen entsprechenden Zahn-Gaumenlaut sprechen, also z.B. partja 'sein Ufer': partya, áldjon 'er mügr segnen' : álgyon, fonjunk 'spinnen wir': fonnyumk.

Nach der Art der Artikulation sind die ungarischen Konsonanten gleichfalls in sechs Reihen einzuteilen. Unter den sechs haben aber drei (das laterale l, der Rollaut $r$ und die Nasalen $m, n, n y)$ in grossem Masse vokalischen Charakter, können also mit jeden anderen Konsonanten in Verbindung treten, ohne dass die sprechenden und die Zuhörer bei der Lautbildung bzw. bei der Kenntnisnahme der Lautbildung Anstoss daran nähmen. So ist die Zahl der Reihen nach der Artikulation der Konsonanten auf drei herabzusetzen. Darunter bilden zwei Reihen, die Verschluss- und die Reibelaute, einen Gegensatz zueinander, die dritte Reihe, die der Verschluss-Reibelaute (Affrikata) ist aber gerade aus der Auflösung und Ausgleichung dieses Gegensatzes hervorgegangen. Daraus folgt logisch, dass die Aussprache den Gegensatz in der unmittelbaren Verbindung eines Verschluss- und Reibelautes durch Verschmelzung auflöst. Die Verschmelzung besteht darin, dass wir anstatt der Verbindung eines dentalen Verschlusslautes und eines an der gleichen Stelle gebildeten Reibelautes einen neuen Laut formen: 
den entsprechenden Verschluss-Reibelaut, der das Moment des Veschlusses und das der Reibung zu einer unzertrennbaren Einheit verschmilzt. So z.B. in látsz 'du siehst' : lácc, tartson 'er möge halten' : tarcson.

\section{Der Formenbau}

3. Nominalflexion. ${ }^{1}$ Der auffallendste Zug der ungarischen Nominalflexion im Vergleich zu anderen Sprachen besteht in der grossen Zahl der Kasus. Gegenüber den fünf Kasus des Isateinischen hat die ungarische Nominalflexion des Substantivs 23 Kasus aufzuweisen. Das Paradigma des Substantivs asztal 'Tisch' z.B. wird auf folgende Weise gebildet (Tabelle 4):

Tabelle 4.

\begin{tabular}{|c|c|c|}
\hline & Name des Kasus & Beispie] \\
\hline 1 & Nominativ & asztal 'der Tisch' \\
\hline 2 & Akkusativ & aszlalt 'den 'Tisch' \\
\hline 3 & Genitiv & asztal(nak) vmije 'des Tisches' \\
\hline 4 & Inessiv & asztalban 'in dem Tisch' \\
\hline 5 & Elativ & asatalból 'aus dem 'Tisch' \\
\hline 6 & Illativ & asztalba 'in den Tisch' \\
\hline 7 & Superessiv & asztalon 'auf dem 'Tisch' \\
\hline 8 & Delativ & asztalról'von dem Tisch' \\
\hline 9 & Sublativ & asztalra 'auf den 'Tisch' \\
\hline 10 & Adessiv & asatalnál 'bei dem Tisch' \\
\hline 11 & Ablativ & asztaltól 'von dem Tisch weg' \\
\hline 12 & Allativ & asztalhoz 'zu dem 'Tisch' \\
\hline 13 & 'T'erminativ & asztalig 'his zum Tisch' \\
\hline 14 & I)ativ & asztalnak 'dem Tisch' \\
\hline 15 & Temporal & (karácsonykor 'zu Weihnachten') \\
\hline 16 & Formal & asztalképp(en) 'als Tisch' \\
\hline 17 & Essiv-Formal & asztalként 'wie ein 'Tisch' \\
\hline 18 & Essiv-Modal & asstalul 'als Tisch' \\
\hline 19 & Distributiv & asztalonként 'nach jedem 'Tisch' \\
\hline 20 & Faktiv-Translativ & asztallá 'zum 'Tisch' \\
\hline 21 & Instrumental-Komitativ & asztallal 'mit dem 'Tisch' \\
\hline 22 & Soziativ & asztalostul 'mit dem Tisch zusammen' \\
\hline 23 & Kausal-Final & asztalért 'um den 'Tisch' \\
\hline
\end{tabular}

${ }^{1}$ Diese Frage hat zuletzt Józse Fompa behandelt in seinem Aufsatz "A magyar névszói ragozásrendszer áttekintése" (Übersicht des ungarischen nominalen Flexionssystems; A Magyar Nyelv és Irodalom Tanítása. Jahrg. II, Nr. 2-3, S. 18-24!. 
Freilich verdoppelt sich die Zahl der Kasusformen, wenn wir in Betracht ziehen, dass fast jeder Kasus auch eine Mehrzahl hat. Ausserdem gibt es im Ungarischen neben der Kasusflexion auch eine possessive Personalflexion und jede mit Possessivendung versehene Form kann dekliniert werden, d.h. jeder Possessivstamm kann die verschiedenen Kasusendungen annehmen. Noch verwickelter wird das System der Nominalflexion dadurch, dass es im Ungarischen ein sog. Besitzzeichen gibt, $-\dot{e}$, welches den Namen des Besitzers zum Namen des Besitzes gestaltet und nicht nur dem Grundwort oder dessen einfachem Mehrzahlstamm angefügt werden kann, sondern auch jeder Form mit Possessivendung. Die Formen mit dem Besitzzeichen - $\dot{e}$ können auch mit dem Pluralzeichen $-i$ pluralisiert werden. Jede Form mit Besitzzeichen kann dann noch nach Belieben mit Kasusendungen versehen werden. Im Flexionssystem des ungarischen Substantivs können also der Kasusflexion 42 Wortformen zugrunde liegen gegenüber zwei Formen des Lateinischen (Einzahl und Mehrzahl). Diese Grundformen gestalten sich bei der Deklination des Wortes asztal 'Tisch' folgendermassen (Tabelle 5):

Tabelle 5.

\begin{tabular}{|c|c|}
\hline \multicolumn{2}{|r|}{ I Absolute Formen } \\
\hline 1 & asztal 'der 'Tisch' \\
\hline 2 & aszalalé 'das des Tisches' \\
\hline 3 & asztaléi 'die des 'Tisches' \\
\hline 4 & asztalok 'Tische' \\
\hline 5 & asztaloké 'das der Tische' \\
\hline 6 & asztalokei $i$ 'die der 'Tische' \\
\hline \multicolumn{2}{|r|}{ II Possessive Formen } \\
\hline 7 & asztalom 'mein Tisch' \\
\hline 8 & asztalomé 'das meines Tisches' \\
\hline 9 & asztalomei 'die meines Tisches' \\
\hline 10 & asztalaim 'meine Tische' \\
\hline 11 & asztalaimé 'das meiner Tische' \\
\hline 12 & asztalaméi 'die meiner Tische' \\
\hline
\end{tabular}




\begin{tabular}{|c|c|}
\hline 13 & asztalod 'dein Tisch' \\
\hline 14 & asztalodé 'das deines Tisches' \\
\hline 15 & asztalodèi 'die deines Tisches' \\
\hline 16 & asztalaid 'deine Tische' \\
\hline 17 & asztalaide' 'das deiner Tische' \\
\hline 18 & asztalaidéi 'die deiner Tische' \\
\hline 19 & asztala 'sein/ihr 'Tisch' \\
\hline 20 & asztaláé 'das seines/ihres Tisches' \\
\hline 21 & asztaláei 'die seines/ihres Tisches' \\
\hline 22 & asztalai 'seine/ihre Tische' \\
\hline 23 & asztalaié 'das seiner/ihrer 'Tische' \\
\hline 24 & asztalaiei 'die seiner/ihrer 'Tische' \\
\hline 25 & asztalunk 'unser 'Tisch' \\
\hline 26 & asztalunké 'das unseres Tisches' \\
\hline 27 & asztalunkéi 'die unseres Tisches' \\
\hline 28 & asstalaink 'unsere Tische' \\
\hline 29 & asztalainké 'das unserer Tische' \\
\hline 30 & asztalainkéi 'die unserer 'Tische' \\
\hline 31 & asztalotok 'euer 'T'isch' \\
\hline 32 & asztalotoké 'das eueres Tisches' \\
\hline 33 & asztalotokẻi 'die eueres Tisches' \\
\hline 34 & asztalaitok 'euere 'Tische' \\
\hline 35 & asztalaitoké 'das euerer Tische' \\
\hline 36 & asztalaitokéi 'die euerer 'Tische' \\
\hline 37 & asztaluk 'ihr Tisch' \\
\hline 38 & asztaluke 'das ihres Tisches' \\
\hline 39 & asztalukéi 'die ihres Tisches' \\
\hline 40 & asztalaik 'ihre Tische' \\
\hline 41 & asztalaiké 'das ihrer Tische' \\
\hline 42 & asztalaikéi 'die ihrer Tische' \\
\hline
\end{tabular}

Zwei Kasusendungen, die des Distributivs -nként und die des Soziativs -stul/-stül, können nur an den blossen Wortstamm treten, daher haben von den 42 Grundformen 41 Formen 21 Kasusformen, eine hingegen 23. Das Endergebnis ist, dass im Ungarischen das Flexionssystem eines einzigen Substantivs 884 Wortformen enthält. Freilich muss man wissen, dass diese 
Zahl nur ein Rahmen ist, nur Möglichkeiten andeutet. Wird doch die Kombination von Wörtern und Endungen nicht einmal durch Bedeutungshindernisse unmöglich gemacht. Ferner wirken manche Wortformen auch sonst künstlich, weil sie in der wirklichen Rede nur selten vorkommen. Doch wenn wir in den 884 Formen mit Endungen selbst nur Möglichkeiten sehen, ist diese Zahl verblüffend gross gegenüber den $2 \times 5=10$ Kasusformen des Lateinischen.

Der auffallend grosse Formenreichtum der ungarischen Sprache ist im Endergebnis das Resultat der synthetischen Natur dieser Sprache. Das Ungarische pflegt nämlich Vorstellungen und Verhältnisse in viel höherem Masse in eine Wortform unter Anhäufung von Suffixen zu integrieren, als die Sprachen mit analytischem Charakter dies tun.

Schon die einfache Flexion mit possessiver Personalendung ist gegenüber der entsprechenden Ausdrucksweise der indoeuropäischen Sprachen eine sehr gedrängte Konstruktion. Dir ungarische Form kezem 'meine Hand' z.B. fasst das Besitzverhältnis des Begriffes 'Hand' und des 'ich' in ein Wort zusammen, während das deutsche meine IIand denselben 'Tatbestand mit einer Konstruktion von zwei Wörtern ausdrückt. Im Ungarischen können aber, wie wir gesehen haben, die Wortformen mit Possessivendung auch noch Kasusendungen annehmen;

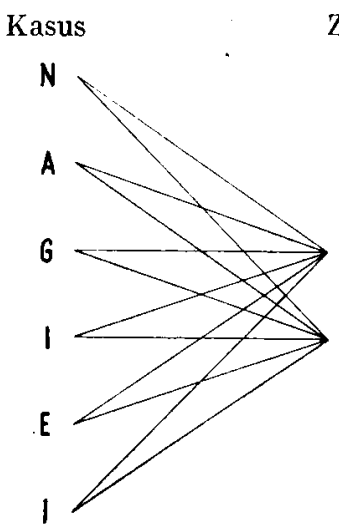

Zahl

Person

Kasus

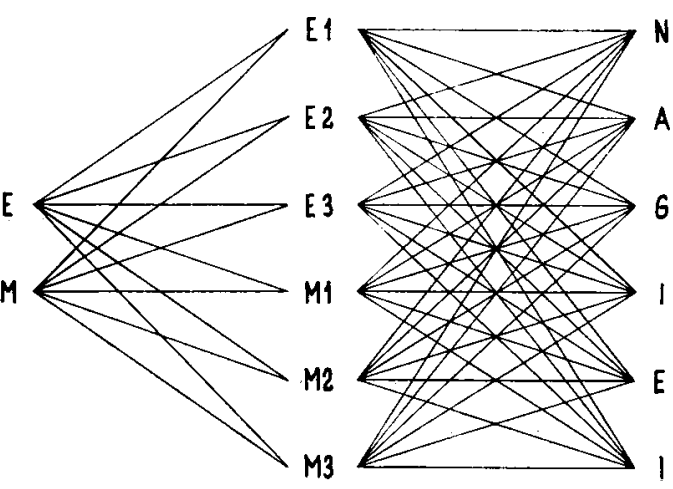

Figur 1. 
eine solche Wortform zeigt natürlich eine noch mehr gedrängte Ausdrucksweise. Wir können es hier nicht unternehmen, die Verdichtungskonstruktion des ganzen ungarischen Deklindtionssystems vorzulegen, und wollen nur das Konstruktionsbild eines engeren Ausschnitts als Beispiel vorführen. Wir gehen davon aus, dass es im Ungarischen eine absolute und eine possessive Deklination gibt. Von den 23 bzw. 21 Kasus nehmen wir jetzt nur 6 als Beispiel ins Schema anf. Aus Raummangel lassen wir auch die Betrachtung der Formen mit dem Besitzzeichen -é im System der Deklination ausser acht. Auf diese Weise erhalten wir vom ungarischen Deklinationssystem folgendes rereinfachte's Schema (Firur 1 und Tabelle 6):

Tabelle 6.

\begin{tabular}{|c|c|}
\hline \multicolumn{2}{|r|}{ I Absolute Deklination } \\
\hline $\begin{array}{l}\text { E-N } \\
\text { E-A } \\
\text { E-G } \\
\text { E-I } \\
\text { E-E } \\
\text { E-I }\end{array}$ & $\begin{array}{l}\text { kéz '(die) Hand' } \\
\text { kezet '(die) Hand' } \\
\text { kéz(nek)'(der) Hand' } \\
\text { kézben 'in (der) Hand' } \\
\text { kézböl 'aus (der) Hand' } \\
\text { kézbe 'in (die) Hand' }\end{array}$ \\
\hline $\begin{array}{l}M-N \\
M-A \\
M-G \\
M-I \\
M-E \\
M-I\end{array}$ & $\begin{array}{l}\text { kezek '(die) Hände' } \\
\text { kezeket '(die) Hände' } \\
\text { kezek(nek) '(der) Hände' } \\
\text { kezekben 'in (den) Händen' } \\
\text { kezekbôl 'aus (den) Händen' } \\
\text { kezekbe 'in (die) Hände' }\end{array}$ \\
\hline \multicolumn{2}{|r|}{ II Possessive leklination } \\
\hline $\begin{array}{l}\text { E-E1-N } \\
\text { E-E1-A } \\
\text { E-E1-G } \\
\text { E-E1-I } \\
\text { E-E1-E } \\
\text { E-E1-I }\end{array}$ & $\begin{array}{l}\text { kezem 'meine Hand' } \\
\text { kezemet 'meine Hand' } \\
\text { kezemnek 'meiner Hand' } \\
\text { kezemben 'in meiner Hand' } \\
\text { kezemböl 'aus meiner Hand' } \\
\text { kezembe 'in meine Hand' }\end{array}$ \\
\hline
\end{tabular}




\begin{tabular}{|c|c|}
\hline $\begin{array}{l}\text { E-E2-N } \\
\text { E-E2-A } \\
\text { E-E2-G } \\
\text { E-E2-I } \\
\text { E-E2-E } \\
\text { E-E2-I }\end{array}$ & $\begin{array}{l}\text { kezed 'deine I Iand' } \\
\text { kezedet 'deine Hand' } \\
\text { kezednek 'deiner Hand' } \\
\text { kezedben 'in deiner Hand' } \\
\text { kezedböl 'aus deiner IIand' } \\
\text { kezedbe 'in deine IIand' }\end{array}$ \\
\hline $\begin{array}{l}\text { E-E3-N } \\
\text { E-E3-A } \\
\text { E-E3-C: } \\
\text { E-E3-I } \\
\text { E-E3-E } \\
\text { E-E3-I }\end{array}$ & $\begin{array}{l}\text { keze 'seine/ihre Hand' } \\
\text { kezét 'seine/ihrt Hand' } \\
\text { kezének 'seinerihrer Hand' } \\
\text { kezében 'in seiner/ihrer Hand' } \\
\text { kezéböl 'aus seiner/ihrer Iand' } \\
\text { kezébe 'in seine/ihre Hand' }\end{array}$ \\
\hline $\begin{array}{l}\text { E-M1-N } \\
\text { E-M1-A } \\
\text { E-M1-G } \\
\text { E-M1-I } \\
\text { E-M1-E } \\
\text { E-M1-I }\end{array}$ & $\begin{array}{l}\text { kezünk 'unsere Iand' } \\
\text { kezünket 'unsere IIand' } \\
\text { kezünknek 'unserer Hand' } \\
\text { kezünkben 'in unserer Iand' } \\
\text { kezünkböl 'aus unserer Hand' } \\
\text { kezünkbe 'in unsere Hand' }\end{array}$ \\
\hline $\begin{array}{l}\text { E-M2-N } \\
\text { E-M2-A } \\
\text { E-M2-G } \\
\text { E-M2-I } \\
\text { E-M2-E } \\
\text { E-M2-I }\end{array}$ & $\begin{array}{l}\text { kezetek 'euere Hand' } \\
\text { kezeteket 'euere IIand' } \\
\text { kezeteknek 'euerer IIand' } \\
\text { kezetekben 'in euerer IIand' } \\
\text { kezetekböl 'aus euerer IIand' } \\
\text { kezetekbe 'in euere IIand' }\end{array}$ \\
\hline $\begin{array}{l}\text { E-M3-N } \\
\text { E-M3-A } \\
\text { E-M3-G } \\
\text { E-M3-I } \\
\text { E-M3-E } \\
\text { E-M3-I }\end{array}$ & $\begin{array}{l}\text { kezük 'ihre Hand' } \\
\text { kezüket 'ihre Hand' } \\
\text { kezüknek 'ihrer Hand' } \\
\text { kezükben 'in ihrer Hand' } \\
\text { kezükböl 'aus ihrer Hand' } \\
\text { kezükbe 'in ihre Hand' }\end{array}$ \\
\hline $\begin{array}{l}\text { M-E1-N } \\
\text { I-E1-I } \\
\text { M-E1-G } \\
\text { M-E1-1 } \\
\text { M-E1-E } \\
\text { M-E1-I }\end{array}$ & $\begin{array}{l}\text { kezeim 'meine IIände' } \\
\text { kezeimet. 'meine Hände' } \\
\text { kezeimnek 'meiner Ilände' } \\
\text { kezeimben 'in meinen IIänden' } \\
\text { kezeimbäl 'aus meinen Händen' } \\
\text { kezeimbe 'in meine Iände' }\end{array}$ \\
\hline
\end{tabular}




\begin{tabular}{|l|l|}
\hline M-E2-N & $\begin{array}{l}\text { kezeid 'deine Hände' } \\
\text { kezeidet 'deine Hände' } \\
\text { M-E2-G } \\
\text { M-Ezeidnek 'deiner IIände' } \\
\text { Mezeidben 'in deinen Händen' } \\
\text { Mezeidböl 'aus deinen IIänden' } \\
\text { M-E2-I }\end{array}$ \\
\hline kezeidbe 'in deine IIände'
\end{tabular}

Die absolute Deklination zeigt das Bild der einfachen Verdichtung: die Verbindung zweier Beziehungspunkte, d.h. zweier Vorstellungen wird in einer einheitlichen Wortform verdichtet. So drückt z.B. die Form kézben 'in der Hand' die Beziehung des Körperteils 'Hand' und des 'Drinnenseins' aus. Die analytische deutsche Sprache drückt dieselbe Beziehung durch die 
Verbindung zweier Wörter aus: in Hand. Die Formen der possessiven Deklination zeigen das Bild der doppelten Verdichtung: hier sind drei Beziehungspunkte, d.h. drei Vorstellungen, bzw. die zwischen ihnen bestehende zweifache Beziehung verdichtet. Z.B. die Wortform kezemben 'in meiner Hand' drückt die Vorstellung des Körperteils 'Hand aus, dann die Personvorstellung 'ich' und die Ortsvor'stellung des 'Darinnenseins'. Bemerkenswert ist, dass die deutsche sprache dieselbe doppelte. Beziehung (das Besitzverhältnis und das Ortsverhältnis) durch die Verbindung dreier Wörter, durch ein dreigliedriges Wortgefüge ausdrückt: in meiner $H$ and.

4. Konjugation. Auf den ersten Blick scheint die Konjugation der ungarischen Sprache ärmlicher zu sein als das Konjugationssystem der bekannten indoeuropäischen Sprachen. Es gibt zwar auch im Ungarischen neben der aktiven Konjugation eine passive, doch ist ihr Gebrauch sehr beschränkt. Noch auffallender ist, dass die moderne ungarische Gemeinsprache nicht mehrere Präteritalformen kennt und für den Ausdruck der Vergangenheit nur cin einfaches Präteritum auf -t gebraucht. Demgegenüber hat aber die ungarische Sprache in bezug auf die Personalflexion ein reiches Formensystem entwickelt, mit dem sie der Konjugation der bekannteren sprachen nicht nachsteht. In der ungarischen Sprache gibt es nämlich zwei Konjugationen: eine subjektive und eine objektive. Z.B. kann das Deutsche mit der Personalendung des Verbs nur die Person und Zahl des Subjekts nennen, während das Ungarische ausser diesen beiden in gewissem Rahmen auch die Person des Objekts zu bezeichnen vermag.

Die Formen der subjektiven oder absoluten Konjugation bezeichnen das Subjekt-Prädikatsverhältnis der verbalen Handlung und der handelnden Person. Die objektive Form drückt hingegen drei Vorstellungen in einer Wortform aus: die der Handlung, der handelnden und der leidenden Person (Subjektperson - Objektperson), bzw. zwei Beziehungen: rine prädikative und eine objektive. So enthält z.B. die subjektive Form cársz 'du wartest' das Subjekt-Prädikatsverhältnis der Handlungsvorstellung des 'Wartens' und der Personalvorstellung 

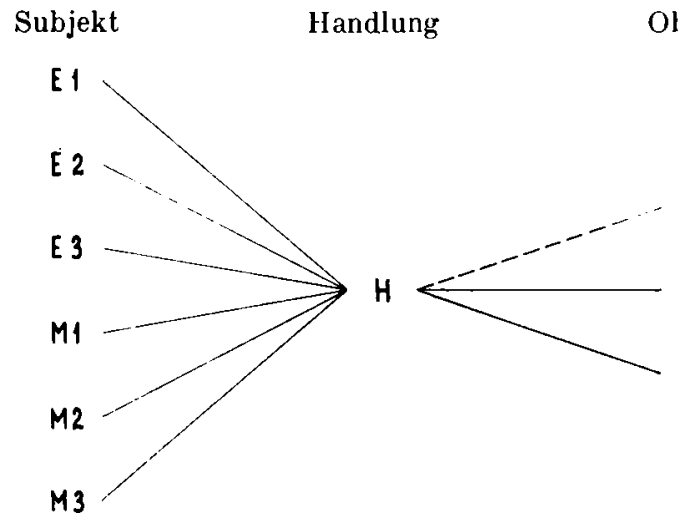

Objekt

Subjekt

Figur 2.

'du', also das, was die deutsche Sprache gewöhnlich mit einer zweigliedrigen Wortkonstruktion ausdrückt: $d u$ wartest. Hingegen verdichtet die objektive Form várod 'du erwartest ihn/ sie/es' drei Vorstellungen: die des 'Wartens', der handelnden Person 'du' und der leidenden Person (oder des Gegenstandes) 'er/sie/es', ferner zwei Beziehungen: die handelnde ('du wartest') und die leidende ('er/sie/es wird erwartet') prädikative Beziehung. Das Deutsche drückt dieses zweifache Verhältnis durch eine dreigliedrige Wortkonstruktion aus: du eruartest ihn/sie/es.

Wenn wir uns aus Gründen der Einfachheit und der Übersichtlichkeit nur auf das Präsens des Indikativs beschränken, können wir das Bild der subjektiven und objektiven Konjugation folgendermassen veranschaulichen (Figur 2 und Tabelle 7 ).

Wie das Strukturbild zeigt, werden in der objektiven Konjugation von den $3 \times 6=18$ Formennöglichkeiten nur 7 so verwirklicht, dass wir die betreffenden Beziehungen mit einer besonderen Verbalform ausdrücken können. Wir können auf diese Weise die Verbindung eines Objekts dritter Person mit dem Subjekt einer jeden Person, ferner die Verbindung eines Objekts zweiter Person mit einem Subjekt erster Person der Einzahl ausdrücken. Für die übrigen Beziehungen stehen uns nur zweigliedrige Wortkonstruktionen zur Verfügung. In die- 
Tabelle 7.

\begin{tabular}{|c|c|}
\hline \multicolumn{2}{|r|}{ I Subjektive Konjugation } \\
\hline $\begin{array}{l}\text { H-E1 } \\
\text { H-E2 } \\
\text { H-E3 } \\
\text { H-M1 } \\
\text { H-M2 } \\
\text { H-.I3 }\end{array}$ & $\begin{array}{l}\text { nézek 'ich schaue' } \\
\text { nézel 'du schaust' } \\
\text { néz 'er schaut' } \\
\text { nézünk 'wir schauen' } \\
\text { néztek 'ihr schaut' } \\
\text { néznek 'sie schauen' }\end{array}$ \\
\hline \multicolumn{2}{|r|}{ Il Objektive Konjugation } \\
\hline $\begin{array}{l}\mathrm{H}-1-\mathrm{E} 1 \\
\mathrm{II}-1-\mathrm{E} 2 \\
\mathrm{H}-1-\mathrm{E} 3 \\
\mathrm{H}-1-\mathrm{M} 1 \\
\mathrm{H}-1-\mathrm{M} 2 \\
\mathrm{H}-1-\mathrm{M} 3\end{array}$ & $\begin{array}{l}\text { (nézem magamat 'ich schaue mich') } \\
\text { (nézel engem/minket 'du schaust mich/uns') } \\
\text { (néz engem/minket 'er schaut mich/uns') } \\
\text { (nézzük magunkat 'wir schauen uns') } \\
\text { (néztek engem/minket 'ihr schaut mich/uns') } \\
\text { (néznek engem/minket 'sie schauen mich/uns') }\end{array}$ \\
\hline $\begin{array}{l}\mathrm{H}-2-\mathrm{E} 1 \\
\mathrm{H}-2-\mathrm{E} 2 \\
\mathrm{H}-2-\mathrm{E} 3 \\
\mathrm{H}-2-\mathrm{MI} \\
\mathrm{H}-2-\mathrm{M} 2 \\
\mathrm{H}-2-\mathrm{M} 3\end{array}$ & $\begin{array}{l}\text { nézlek 'ich schaue dich' } \\
\text { (nézed magadat 'du schaust dich') } \\
\text { (néz téged/titeket 'er schaut dich/euch') } \\
\text { (nézünk téged/titeket 'wir schauen dich/euch') } \\
\text { (nézitek magatokat 'ihr schaut euch') } \\
\text { (néznek téged/titeket 'sie schauen dich/euch') }\end{array}$ \\
\hline $\begin{array}{l}\mathrm{H}-3-\mathrm{E} 1 \\
\mathrm{H}-3-\mathrm{E} 2 \\
\mathrm{H}-3-\mathrm{E} 3 \\
\mathrm{H}-3-\mathrm{M} 1 \\
\mathrm{H}-3-\mathrm{M} 2 \\
\mathrm{H}-3-\mathrm{M} 3\end{array}$ & 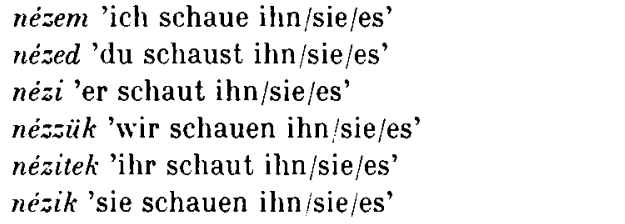 \\
\hline
\end{tabular}

sen Wortgefügen verbinden wir gewöhnlich die entsprechende Verbform des Subjekts mit der Objektform des entsprechenden Personalpronomens. Wenn aber die Person des Subjekts mit der des Objekts übereinstimmt, dann wählt man zum Ausdruck einer solchen Beziehung die Verbindung der objektiven Form des Verbs mit der entsprechenden Form des reflexiven Pronomens. 


\section{Der Saztbau}

5. Parataktische Konstruktionen. Parataktisch nennt man eine Konstruktion, deren Glieder zu irgendeinem andern Glied des Satzes im gleichen Verhältnis stehen, eben deswegen einander gleichgestellt sind. Der formale Ausdruck der Parataxe ist eine Kongruenz, die darin besteht, dass man die Glieder mit denselben Endungen, eventuell mit der Ø-Stufe der Endungen versieht. Der Gebrauch derselben Endung zeigt, dass die Glieder in gleicher Weise von einem aussenstehenden Glied abhängig sind, ausserdem wird aber dadurch die Gleichgestelltheit der parataktischen Glieder ausgedrückt. Z.B. éjtnapot eggyé tett 'er vereinigte Nacht und Tag': in diesem Satze finden wir ein zweigliedriges Objekt vor: éjt und napot. Die Endung des Objekts - $t$ deutet ron beiden Gliedern auf den verbalen Teil eggyé tett hin, ausserdem drückt sie aber auch die Zusammengehörigkeit, Gleichgestelltheit, manchmal auch die Parallelität der Glieder aus.

Die parataktischen Glieder bilden im allgemeinen eine offene Reihe. Eine solche ist z.B. okos, liedves, bájos teremtés 'ein kluges, liebes, anmutiges Geschöpf', wo man die syntaktisch gleichgestellten Attribute noch nach Belieben vermehren könnte. Doch gibt es auch solche parataktische Verbindungen, die notwendigerweise zweigliedrig sind, wo die Glieder aber etwas Ganzes, zwei Seiten einer Sache, zwei Aspekte bezeichnen. Das sind die sprachlichen Parallelen. Die parallele Konstruktionsart ist eine altertümliche sprachliche Eigentümlichkeit. Ihr Ausdrucksmittel ist die Wiederholung der Endungen, mit anderen Worten die Kongruenz.

Die ungarische Sprache ist voll von parallelen Ausdrücken, die an uralte Zeiten erinnern. Um nur aufs Geratewohl einige zu nennen: éjt-napot eggyé tett 'er vereinigte Tagr und Nacht; éjjelnappal dolgozott 'er arbeitete 'Tag und Nacht'; hegyen-völgyön lakodalom 'Hochzeit auf Berg und 'Tal'; árkon-bokron túl volt 'er war über Stock und Stein'; ágas-bogas fa 'ein reichverzweigter Baum'; sütött-fözött egész nap 'sie kochte und buk den ganzen 'Tag'; sokat lótott-futott 'er lief viel hin und her'; hetet-havat összehord 'er trägt alles mögliche zusammen'; elállt 
szeme-szája 'er sperrte Ilund und Augen auf'; fũnek-fának elmesélte 'er erzählte es aller Welt'; égre-földre eskï̈dözönl 'er schwor auf Himmel und Erde'; orrán-száján eleredt a vér 'das Blut floss ihm aus Mund und Nase'; télen-nyáron pusztán az ín lakisom 'im Winter und Sommer ist meine Wohnung auf der Puszta'; hébe-hóba hazalátogatott 'dann und wann kam er' nach Hause'; ez csak olyan mendemonda 'das ist nur solches Gerede'; nagy hercehurca volt az 'das war eine grosse Schererei'; csillog-villog jó vitéz fegyrere 'es glänzt und blitzt seine gute Heldenwaffe'; sokat izgett-mozgott 'er bewegte sich hin und her'; csak térül-fordul 'er kehrt und windet sich'; folyton eszik-iszik 'er isst und trinkt in einem fort'; leng-lobog a magyar zászló 'es weht und flattert die ungarische Fahne'; ment, mendegélt 'er ging und wandelte'; jobbra-balra tekinget 'er blickt immer nach rechts und links'; az ilyen-amolyan 'der verdammte Kerl'.

Der parataktische bzw. parallele Ausdruck bildet manchmal eine Einheit nach Art einer Zusammensetzung. Der sprachliche Ausdruck dieser Erscheinung ist, dass nur das letzte Glied Formantien annimmt. Es liegt also keine Kongruenz vor, ebenso wie bei den hypotaktischen attributiven Konstruktionen. Mendemonda und viele andere "Doppelwörter" zeigen zwar die Idlentität der Endung in beiden Gliedern, die weiteren Beziehungselemente schliessen sich aber nur einmal dem ganzen Ausdruck an: sok mendemondát hallani. Auch bei anderen, deutlicher gegliederten Parallelen kommt es vor, dass das Akzessionselement nur einmal angefügt wird, z.B. szemszájnak ingere 'ein Reiz für Mund und Augen' anstatt szemnek-szájnak.

6. Hypotaktische Konstruktionen. In den hypotaktischen Konstruktionen gibt es ein Hauptglied und eine untergeordnete Ergänzung. Die drei Hauptarten sind: attributive, objektartige und adverbiale Konstruktion.

Die Adjektiv- und Numeralattribute bilden im Ungarischen ein kompositorisches Gefüge mit ihrem Hauptglied. Formantien erhält nur das jeweilige Hauptglied, nicht aber das Attribut, z.B. jó ember 'guter Mensch', jó embert 'guten Menschen'; 
Papp István (Nom.), l'app Istuánnak (1)at.); száz forint 'hundert Forint', száz forintért 'um hundert Forint'. Es kommt jedoch auch vor, dass das Attribut nach seinem Hauptglied steht; bei solchen "Nachstellungen" tritt die Kongruenz auf, z.B. reftem neki csizmat, pirnsat 'ich kanfte ihr Stipfel, rote'; Zrimyivel a költốvel larmml; wir halten es mit Zrínyi, dem Dichter"; madarat nem egyet, szizat is meglönek "sie schiessen nicht einen Vogel, sondern sogar hundert'. Die Konstruktion mit Nachstellung ist eigentlich ein Appositionsverhältnis und steht also in einem gewissen Zusammenhang mit der Parataxe. Demgemäss sind in dem Satze: rettem neli csizmát, pirosat das Hauptglied und sein Attribut parataktische, gleichgestellte Glieder und stehen miteinander in erklärondem Verhältnis: rettem neki csizmit, ti. pirosal ich kaufte ihr Stiefel, nämlich rote'.

Das pronominale Attribut stand vor seinem Hauptglied ursprünglich immer endungslos. So kongruierte in der alten Sprache das Demonstrativum $e z / a z$ als Attribut nicht mit dem Hauptglied: az ember 'jener Mensch', az embernek 'jenem Menschen'. Heute wird die Erinnerung an diese alte Konstruktionsart nur noch in einigen feststehenden Ausdrücken bewahrt, wie z.B. ez alkalommal 'bei dieser Gelegenheit', ezúttal 'diesmal', e n'pnek 'diesem Volk', ama naptól fogva 'von jenem Tag an'. Sonst bleibt das pronominale Attribut auch heute undekliniert, z.B. nem tudom, mi fán terem 'ich weiss nicht, auf welchem Baum es wächst ( $=$ was es eigentlich ist)'. Hingegen kongruiert das demonstrative az/ez als Attribut gewöhnlich mit seinem Hauptrrlied: ez/az az ember 'dieser/jener Mensch'. ezt/azt az embert 'diesen/jenen Menschen', ennek/annak az embernek 'diesem/jenem Menschen'. Diese Form der Kongruenz drückte ursprünglich ein Appositionsverhältnis aus, nähert sich also seinem Wesen nach dem parataktischen Verhältnis: ezt, tudniillik az embert várom 'den, nämlich den Menschen erwarte ich'.

Im Ungarischen haben sich drei Formen der possessiven Konstruktion entwickelt. Eine uralte Ausdrucksweise enthalten die Zusammensetzungen mit unbezeichnetem Verhältnis: hiztetö 'Hausdach', tengerpart 'Meeresstrand', wo der Besitzer 
vor dem Besitzwort steht und damit eine synthetische Einheit bildet. Der zweite Formentyp ist der, in dem die Possessivendung des Besitzwortes, des Hauptgliedes der Konstruktion, auf den Besitzer hinweist, wobei letzteres Wort stets vor dem Besitzwort steht, z.B. az Isten haragja 'der Zorn Gottes', a fiu könyre 'das Buch des Knaben'. Sonst ist auch diese Konstruktion ein einheitlicher Ausdruck synthetischer Natur, da weitere Beziehungselemente anzunehmen nur das letzte Glied fähig ist, z.B. a fiú könyvét 'das Buch des Knaben (Akk.)', a fiú lönyviben 'im Buch des Knaben'. Dennoch ist diese Konstruktionsart etwas lockerer als die frühere, und hat ein Element, das auf das parataktische bzw. Appositionsverhältnis hinweist. Die Verbindung az Isten csodája 'das Wunder Gottes' drückt nämlich aus, dass ein vorangestellter Nominativ ausrufsartig für sich steht, vorauf die Konstruktion von neuem beginnt, mit einem Pronomen, das jedoch als possessive Bestimmung in den Satz eingebaut wird: Isten, az ö csodája... 'Gott, sein Wunder...'. Endlich gibt es in der ungarischen Sprache auch eine dritte Form der possessiven Ausdrucksweise: a tengernek a partja 'der Strand des Meeres', a fiúnak a könyje 'das Buch des Knaben'. Hier erfolgt eine zweifache Bezeichnung: die Possessivendung des Besitzwortes weist auf den Besitzer, andererseits zeigt aber die Endung -nak/-nek des Besitzers auf den Besitz. Diese wechselseitige Beziehungsbezeichnung, die sog. Korrelation ist ein eigenartiges Mittel der ungarischen Satzbildung und drückt eine gewisse Parataxe bzw. ein doppeltes Appositionsverhältnis aus. A tengernek a partja 'des Meeres Strand' verdichtet nach seiner syntaktischen Form zwei Appositionsverhältnisse: 1. a tengernek valamije, tudniillik a partja 'des Meeres etwas, d.i. sein Strand'; 2. az $o$, tudniillik a tengernek a partja 'sein, d.i. des Meeres Strand'.

Auch die objektartigen Konstruktionen haben nach ihrem grammatischen Aufbau dreierlei Formen. Die erste ist das unbezeichnete Objekt, die älteste Konstruktionsweise. Hier wird das hypotaktische Verhältnis nur durch synthetische Zusammenfassung, bzw. durch die eingipflige dynamische Ausbildung bezeichnet: favágó 'Holzhauer', gólyavárni 'den Storch erwarten'. Diese Form ist ihrem Wesen nach identisch mit 
der uralten Ausdrucksweise der attributiven Konstruktion, und es ist wahrscheinlich, dass sich dieselbe im Laufe der Entwicklung von der letzteren abgesondert hat'. Der zweite Formentyp ist das bezeichnete Objekt: die Endıng -t weist auf das Hauptglied der Konstruktion, auf das Verb: fát vág 'er hackt Holz', gólyát rárok 'ich erwarte einen storch'. Im dritten Formentyp herrscht Korrelationskonstruktion: das -t des Objekts weist auf das Verb, während die objektive Personalendung des Verbs auf die Person des Objekts zeigt: a fát vágja 'er hackt das Holz', a gólyát várom 'ich erwarte den Storch'. Die Korrelationsform gebraucht man nur im Falle eines bestimmten Objekts und drückt mit dieser Konstruktion ausser dem hypotaktischen Objektsverhältnis auch das Appositionsverhältnis, bzw. das parataktische Verhältnis aus: a fát vágja 'er hackt das Holz' = a fát, azt rágja 'das Holz, das hackt er'.

Dieselben drei Formentypen finden wir auch bei den adverbialen Konstruktionen vor. Die unbezeichnete Bestimmung sonderte sich im Laufe der Entwicklung wahrscheinlich ron der uralten attributiven Konstruktion ab². Heute ist ihr Gebrauch schon selten: mezítláb szaladgál 'er läuft barfuss', vasárnap pihenek 'sonntags ruhe ich'. Häufiger als diese ist die bezeichnete Bestimmung, bei der die Bestimmungsendung der Ergänzung auf das Hauptolied, das Bestimmte hinzeigt: kézen aill 'er steht auf der Hand (macht Handstand)', isholiból jön 'er kommt aus der Schule'. Endlich gibt es auch hier die Korrelationsform, d.i. die wechselseitige Beziehung. Es kommt nämlich vor, dass von der Bestimmung eine Bestimmungsendung auf das Verb zeigt, während andererseits vom Verb ein Präfix, das die gleiche Funktion hat wie die Bestimmung, auf die Bestimmung weist, z.B. kilép a körböl 'er tritt aus dem Kreis (heraus)', beleesik a rizbe 'er fällt (hinein) ins Wasser', otthagyja a földön 'er lässt es (dort) auf der Erde'.

1 I. ANTAL KLEM versuchte unlängst nachzuweisen, dass das endungslose Objekt und das endungslose Adverbiale darauf hindeuten, dass diese Satzteile sich aus dem Attribut entwickelt haben (NyK LXI $45-52)$.

2 Vgl. I. A.vtal Klemi a.a.O. 
7. Prädikative Konstruktionen. Im Ungarischen gibt es dreierlei prädikative Konstruktionen. Auch ein Nomen kann für sich als Prädikat stehen, wenn der Satz in der 3. Person des Präsens Indikativ steht, z.B. a fiú jó 'der Knabe (ist) gut'; a fiúk jók 'die Knaben (sind) gut'. Die wichtigste Formeneigentümlichkeit der rein nominalen prädikativen Konstruktion ist die Numeruskongruenz: das Prädikat kongruiert im Numerus mit dem Subjekt. Zweitens kann als Prädikat ein Verb, bzw. ein finites Verb stehen: te írsz 'du schreibst'; $t i$ irtok 'ihr schreibt'. Im Satz mit verbalem Prädikat besteht Personalund Numeruskongruenz zwischen Subjekt und Prädikat. Endlich können im ungarischen Satz ein Nomen und ein sog.

Tabelle 8.

\begin{tabular}{|c|c|}
\hline \multicolumn{2}{|r|}{ I Ind. Präsens } \\
\hline $\begin{array}{l}\text { E1 } \\
\text { E2 } \\
\text { E3 } \\
\text { M1 } \\
\text { M2 } \\
\text { M3 }\end{array}$ & $\begin{array}{l}\text { (en) jo vagyok 'ich bin gut' } \\
\text { (te) jó vagy 'du bist gut' } \\
\text { a fiù jo 'der Knabe (ist) gut' } \\
\text { (mi) jok vagyunk 'wir sind gut' } \\
\text { (ti) jók vagytok 'ihr seid gut' } \\
\text { a fiúk jok 'die Knaben (sind) gut' }\end{array}$ \\
\hline \multicolumn{2}{|r|}{ II Ind. Imperfekt } \\
\hline $\begin{array}{l}\mathrm{E} 1 \\
\mathrm{E} 2 \\
\mathrm{E} 3 \\
\mathrm{M} 1 \\
\mathrm{M} 2 \\
\mathrm{M} 3\end{array}$ & $\begin{array}{l}\text { (én) jó voltam 'ich war gut' } \\
\text { (te) jó voltál 'du warst gut' } \\
\text { a fiú jó volt 'der Knabe war gut' } \\
\text { (mi) jok coltunk 'wir waren gut' } \\
\text { (ti) jók roltatok 'ihr waret gut' } \\
\text { a fiúk jók coltak 'die Knaben waren gut' }\end{array}$ \\
\hline \multicolumn{2}{|r|}{ Il I Kond. Präsens } \\
\hline $\begin{array}{l}\text { E1 } \\
\text { E2 } \\
\text { E3 } \\
\text { M2 } \\
\text { M3 }\end{array}$ & $\begin{array}{l}\text { (én) jo volnék 'ich wäre gut' } \\
\text { (te) jó volnál 'du wärest gut' } \\
\text { a fiù jo volna 'der Knabe wäre gut' } \\
\text { (mi) jók volnánk 'wir wären gut' } \\
\text { (ti) jók volnátok 'ihr wäret gut' } \\
\text { a fiúk jók volnának 'die Knaben wären gut' }\end{array}$ \\
\hline
\end{tabular}


Hilfsverb ein zusammengesetztes Prädikat bilden, z.B. te jo vagy 'du bist gut'; ti jók vagytok 'ihr seid gut'. In diesem Falle schliesst sich das nominale Prädikat mit Numeruskongruenz an das Subjekt an, das verbale Prädikat hingegen mit Numerus- und Personenkongruenz. Eine gemeinsame Eigentümlichkeit aller prädikativen Konstruktionen ist der besondere $\mathrm{Ak}$ zent des Subjekts und Prädikats, also die zweigipflige dynamische Ausbildung. Der verbale Teil des zusammengesetzten Prïdikats - die Kopula - schliesst sich entweder an den nominalen Teil des Prädikats oder an das Subjekt an: $t e-j o$ vagy oder te ragy - jó.

Paradigmatisch zusammengestellt ergänzt das zusammen-

Tabelle 9.

I Ind. Präsens

\begin{tabular}{|c|c|}
\hline E1 & (én) irok 'ich schreibe' \\
\hline E2 & (te) irsz 'du schreibst' \\
\hline E3 & a fiú ir 'der Knabe schreibt' \\
\hline M1 & (mi) irunk 'wir schreiben' \\
\hline $\mathrm{M} 2$ & (ti) irtok 'ihr schreibt' \\
\hline M3 & a fiük irnak 'die Knaben schreiben' \\
\hline \multicolumn{2}{|r|}{ II Ind. Imperfekt } \\
\hline $\mathbf{E} 1$ & (én) irtam 'ich schrieb' \\
\hline $\mathrm{E} 2$ & (te) irtál 'du schriebst' \\
\hline E3 & a fiu irt 'der Knabe schrieb' \\
\hline M1 & (mi) irtunk 'wir schrieben' \\
\hline M2 & (ti) irtatok 'ihr schriebt' \\
\hline M3 & a fiuk irtak 'die Knaben schrieben' \\
\hline \multicolumn{2}{|r|}{ III Kond. Präsens } \\
\hline E1 & (én) irnék 'ich würde schreiben' \\
\hline E2 & (te) irnál 'du würdest schreiben' \\
\hline E3 & a fiú irna 'der Knabe würde schreiben' \\
\hline M1 & (mi) irnánk 'wir würden schreiben' \\
\hline $\mathbf{M} 2$ & (ti) irnátok 'ihr würdet schreiben' \\
\hline M3 & a fiuk irnának 'die Knahen würden schreiben' \\
\hline
\end{tabular}


gesetzte Prädikat die Konjugationsmängel des reinen Nominalprädikats (Tabelle 8).

Das Verbalprädikat zeigt natürlich schon für sich ein volles Paradigma (Tabelle 9).

Der Sprachgebrauch hält eben darum nur zwei Haupttypen auseinander und stellt bei den Prädikatsausdrücken rinen nominalen (rein nominal oder verbal-nominal) und einen verbalen Typ nebeneinander. Denselben Tatbestand kann man manchmal auf beide Weisen ausdrücken. Der Unterschied zwischen der nominalen und rerbalen tbart ist nur stilistisch: die nominale Ausdrucksweise ist bündiger und gefühlsbetonter, die rerbale genauer, aber gefühlsmässig gleichgültiger. Vergleichen wir z.B. folgende nominale und verbale Abarten: az Isten a megmondhatója - csak az Isten tudná megmondani 'Crott nur könnte es sagen'; ín a kerek vilíg bujdosója ragyok - bujdosok a kerek világon 'ich wandere auf dieser runden Welt umher'.

İbrigens können wir in allgeneinen feststellen, dass die ungarische Sprache die uralten, primitiven Ausdrucksformen neben den neueren sprachlichen Errungenschaften in grossem Masse bewahrt hat und demzufolge über einen Reichtum von Ausdrucksformen verfügt, der diese Sprache zu einer Nuanciprung der Gedanken befähigt und auch den Ausdruck von Gemütsbewegungen und Willensäusserungen ermöglicht. Und das nimmt im Endergebnis auch nicht wunder, wurde doch die ungarische Sprache Jahrhunderte und .Jahrtausende hindurch von den traditionstrellen, tiefen Schichten der ungarischen Gemeinschaft bewahrt und erhalten, und waren doch andererseits die grossen Persönlichkeiten der Wissenschaft und der Dichtkunst bestrebt, dieselbe den neuen Liedern der neuen Zpit anzupassen. 